\title{
SIMULAÇÃO DE MÓDULOS INDUSTRIAIS DE MEMBRANAS PARA SEPARAÇÃO DE MISTURAS $\mathrm{CO}_{2} / \mathrm{CH}_{4}$
}

\author{
L. S. QUEIROZ ${ }^{1}$, R. A. REIS ${ }^{1}$ e A. L. ALBERTON ${ }^{1}$ \\ ${ }^{1}$ UERJ/Departamento de Operações e Projetos Industriais \\ E-mail para contato: rdosreis@uerj.br
}

\begin{abstract}
RESUMO - A exploração do gás natural oriundo do Pré-Sal, contendo elevada quantidade de $\mathrm{CO}_{2}$, exige tecnologias compactas de separação tal como a permeação de gases por membranas. Neste contexto, o objetivo deste trabalho foi simular em código computacional EMSO e avaliar configurações e modos de operação em estágios formados por módulos de fibras-ocas especificados para remoção de $\mathrm{CO}_{2}$ do gás natural no cenário do Pré-Sal brasileiro. A partir de uma abordagem envolvendo grupos adimensionais e valores típicos de permeabilidade e seletividade em membranas de acetato de celulose e condições operacionais do Pré-Sal, foi possível avaliar a influência do modo de operação em um único estágio e da composição de entrada sobre a área de membrana e a recuperação de metano para atingir uma composição especificada no concentrado. Para ambas variáveis, o modo co-corrente apresentou resultados inferiores, principalmente para menores razões de pressão e teores de $\mathrm{CO}_{2}$ no concentrado. Admitindo mais de um estágio de separação, recirculação de correntes e ciclos de compressão, ficou claro neste estudo que a configuração mais adequada dependerá do destino de cada corrente efluente do sistema de separação.
\end{abstract}

\section{INTRODUÇÃO}

Todo gás natural produzido em plataformas offshore precisa ser tratado de forma a manter sob controle problemas de corrosão, hidratação, incrustação e segurança de manuseio nos gasodutos para escoamento da produção. O dióxido de carbono $\left(\mathrm{CO}_{2}\right)$, um gás quimicamente considerado inerte, quando associado à água livre ocasiona aumento na taxa de corrosão; tanto nos equipamentos de superfície quanto nos dutos de exportação de gás (Engelien, 2004). Além disso, ambientalmente é considerado um dos principais responsáveis pelo efeito estufa (Deng e Hagg, 2010; Nakao et al. 2009; Kundu et al., 2013). Nos campos mais antigos, o gás natural apresenta teores de $\mathrm{CO}_{2}$ em torno de 5\% em volume, nível adequado para o tratamento em torres de absorção com aminas (usualmente monoetanolamina - MEA) (Baker e Lokhandwala, 2008; Gadelha et al., 2012), entretanto, nas jazidas na região denominada de Pré-Sal, os teores médios de $\mathrm{CO}_{2}$ dos campos do Pré-Sal estão sendo encontrados na faixa de $8 \%$ a $45 \%$ em volume. Nestas condições, as vazões circulantes de solução de MEA ficariam muito grandes, obrigando a utilização de equipamentos de porte e peso incompatíveis com o ambiente offshore, além de um enorme gasto de energia para regeneração da amina (Baker e Lokhandwala, 2008). 
Alternativamente, os processos de separação de gases por membranas vêm sendo bastante considerados para a remoção de $\mathrm{CO}_{2}$ do gás natural no cenário do Pré-Sal (Deng e Hagg, 2010; Nakao et al. 2009; Gadelha et al., 2012; Kundu et al., 2013). Trata-se de um processo bastante atrativo para uso offshore, dada a sua simplicidade de operação e manutenção (não possui partes móveis), robustez, baixo peso, baixa demanda de área de montagem e custos atrativos, especialmente em correntes de gás natural com elevado teor de $\mathrm{CO}_{2}$ (Baker e Lokhandwala, 2008; Nakao et al. 2009).

Por outro lado, uma mudança de tecnologia no tratamento primário do gás natural envolveria muitos desafios, tais como, definição de um novo sistema o pré-tratamento do gás para eliminação de $\mathrm{H}_{2} \mathrm{~S}$, umidade e hidrocarbonetos mais pesados e a seleção de: (i) um material adequado para sintetizar as membranas (camada densa de $0,5 \mu \mathrm{m}$ formada por um polímero vítreo ou elastomérico suportada por uma membrana porosa de micro ou ultrafiltração); (ii) de uma estrutura de módulo (espiral ou fibra oca); (iii) e o projeto da unidade industrial, que não somente atenda as especificações das correntes geradas com o menor custo de capital e de operação, mas que também suporte as elevadas pressões envolvidas nesta operação (Scholes et al., 2012, Baker e Lokhandwala, 2008). Devido aos baixos teores dos outros contaminantes, há uma tendência de se utilizar processos de separação de gases por membranas apenas para remoção do $\mathrm{CO}_{2}$ da corrente pré-condicionada do gás natural.

Apesar de diversos polímeros terem se mostrado promissores para este fim (Scholes et al., 2012; Yave et al., 2010), membranas de acetato de celulose são ainda as mais comercialmente utilizadas (Baker e Lokhandwala, 2008). Atualmente, a maioria dos módulos, tanto em espiral ou de fibras ocas, são projetados para caber em caixas cilíndricas de $0,2 \mathrm{~m}$ de diâmetro e $1 \mathrm{~m}$ de comprimento, entretanto módulos de até $0,76 \mathrm{~m}$ de diâmetro estão sendo instalados em algumas unidades (Baker e Lokhandwala, 2008). Recentemente a PNBV, subsidiária da Petrobras, contratou o fornecimento de sistemas de membranas a base de acetato de celuloce para processar gás natural offshore, deixando ainda em aberto, em um primeiro momento, apenas questões envolvendo a configuração das plantas que serão adotadas em suas plataformas do Pré-Sal. Alguns trabalhos da literatura têm explorado tais estratégias tanto neste cenário (Nakao et al. 2009; Gadelha et al., 2012), quanto em outros, tal como no tratamento do biogás (Deng e Hagg, 2010; Kundu et al., 2013). Em particular, o trabalho de Gadelha et al. (2012), indica que uma combinação da tecnologia de membranas combinadas com absorção com aminas apresenta-se como alternativa economicamente promissora para atender as especificações do Pré-Sal brasileiro. Nestes trabalhos, questões envolvendo a utilização de reciclos e sistemas multiestágios na minimização de áreas de membrana (custo de capital), ciclos de compressão e perdas de metano (custo de operação) vem auxiliando na definição das estruturas mais promissoras. A maioria desses trabalhos não fazem uso de códigos computacionais livres, o que, associados a questões de confidencialidade,limitam a disponibilização dos resultados obtidos e a formação de recursos humanos nesta área.

O objetivo principal deste trabalho é, portanto, obter a simulação e avaliação de algumas configurações e modos de operação de separação por membranas na remoção de $\mathrm{CO}_{2}$ do gás natural produzido no cenário do Pré-Sal brasileiro. Para tal, será adotado o código computacional EMSO, que mostra-se vantajoso por ser um código livre para fins acadêmicos 
e de baixo custo para uso industrial, de fácil instalação, que permite total visualização e personalização dos códigos implementados.

\section{MODELAGEM MATEMÁTICA E SIMULAÇÃO}

Cada estágio de separação foi considerado formado por módulos de fibras-ocas esquematicamente ilustrado na Figura 1. Nesta Figura, são também apresentas as principais variáveis adimensionais adotadas na modelagem em um único estágio.

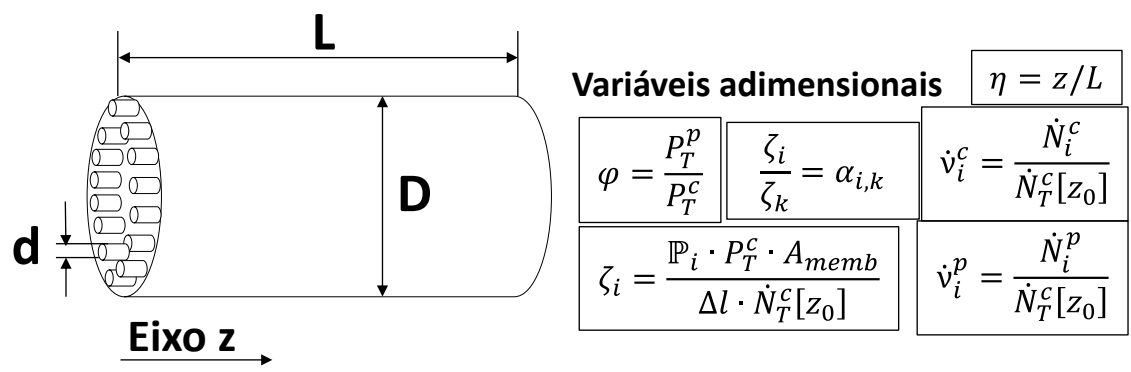

Figura 1 - Representação esquemática do módulo de fibras-ocas e principais grupos adimensionais da modelagem em um único estágio.

Na modelagem de cada estágio de permeação, foram admitidas as seguintes hipóteses:

i. fluxo empistonado tanto no permeado quanto no concentrado, sem perfil de velocidade radial ou influência de dispersão axial;

ii. resistência à transferência de massa externa a pele densa da membrana desprezíveis tanto na corrente de concentrado, quanto na de permeado;

iii. influência da curvatura da fibra e da resistência do suporte poroso à transferência de massa e de momento são desconsiderados no cálculo do fluxo através da membrana;

iv. queda de pressão desprezível ao longo do estágio;

v. as permeabilidades e seletividades dos gases na pele densa da membrana são consideradas constantes para qualquer posição do sistema;

portanto, as equações de balanço material para o concentrado e para o permeado seguem as seguintes expressões, respectivamente:

$$
\underbrace{\frac{d \dot{N}_{i}^{c}}{d z}}_{\begin{array}{c}
\text { Varia cão } \\
\text { da vazão de } i
\end{array}}=-\underbrace{\frac{\mathbb{P}_{i}}{\Delta l} \cdot\left(P_{i}^{c}-P_{i}^{p}\right)}_{\begin{array}{c}
\text { Fluxo que atravessa } \\
\text { a membrana } \\
\text { nocentrado } \text { eixo z }
\end{array}} \cdot \underbrace{N_{\text {fibras }} \cdot \pi \cdot d}_{\begin{array}{c}
\text { Área externa de } \\
\text { membrana por } \\
\text { unidade de } \\
\text { comprimento }
\end{array}} \quad i=\mathrm{CO}_{2}, \mathrm{CH}_{4}
$$

$$
\underbrace{\frac{d \dot{N}_{i}^{p}}{d z}}_{\begin{array}{c}
\text { Varia ção } \\
\begin{array}{c}
\text { a vaz a de } i \\
\text { no permeado } \\
\text { no eixo z }
\end{array}
\end{array}}= \pm \underbrace{\frac{\mathbb{P}_{i}}{\Delta l} \cdot\left(P_{i}^{c}-P_{i}^{p}\right)}_{\begin{array}{c}
\text { Fluxo que atravessa } \\
\text { a membrana }
\end{array}} \cdot \underbrace{N_{\text {fibras }} \cdot \pi \cdot d}_{\begin{array}{c}
\text { Área externa de } \\
\text { membrana por } \\
\text { unidade de } \\
\text { comprimento }
\end{array}} \quad\left(\begin{array}{c}
\text { o sinal depende } \\
\text { do modo: } \\
\text { co }- \text { corrente }(-) \\
\text { contracorrente }(+)
\end{array}\right)
$$


sendo $\dot{N}$ representa a vazão molar, $\mathbb{P}$ a permeabilidade no material da pele densa da fibra, $P$ a pressão, $N_{\text {fibras }}$ o número de fibras, $d$ o diâmetro da fibra, $\Delta l$ a espessura da pele densa da fibra e $L$ é o comprimento do módulo, o sobrescrito $c$ indica concentrado, $p$ permeado, e o subescrito $i$ indica o composto. Os sinais indicam o modo de operação: (-) co-corrente e (+) contracorrente.

\subsection{Resolução dos modos concorrente e contracorrente em único estágio}

Para sistemas em um único estágio, pode-se efetuar o adimensionamento do modelo como segue: (i) as vazões adimensionadas são calculadas tomando-se como referência a vazão molar total alimentada no estágio, i.e. $\dot{v}_{i}^{c}=\dot{N}_{i}^{c} / \dot{N}_{T}^{c}\left[z_{0}\right]$; (ii) o comprimento é adimensionado em relação ao comprimento do estágio, $\eta=z / L$; (iii) define-se um grupo adimensional $\varphi$ sendo a razão entre a pressão total no concentrado e a pressão total no permeado; (iv) grupos adimensionais associados à cada composto $\zeta_{i}$ envolvendo a permeabilidade de cada composto (Figura 1); e (v) a seletividade ideal entre dois compostos $i$ e $k\left(\alpha_{i, k}\right)$ é definida como a razão entre $\zeta_{i} / \zeta_{k}$. Esta abordagem é similar à apresentada em outros trabalhos da literatura (Kundu et al.2013). Em termos das variáveis adimensionais, as equações de balanço ficam:

$$
\begin{aligned}
& \frac{d\left(\dot{v}_{i}^{c}\right)}{d \eta}=-\zeta_{i} \cdot\left(\frac{\dot{v}_{i}^{c}}{\sum_{j=1}^{N C} \dot{v}_{j}^{c}}-\varphi \cdot \frac{\dot{v}_{i}^{p}}{\sum_{j=1}^{N C} \dot{v}_{j}^{p}}\right), \quad i=C O_{2}, C H_{4} \\
& \frac{d\left(\dot{v}_{i}^{p}\right)}{d \eta}= \pm \zeta_{i} \cdot\left(\frac{\dot{v}_{i}^{c}}{\sum_{j=1}^{N C} \dot{v}_{j}^{c}}-\varphi \cdot \frac{\dot{v}_{i}^{p}}{\sum_{j=1}^{N C} \dot{v}_{j}^{p}}\right)
\end{aligned}
$$

A condição inicial das vazões adimensionais alimentadas é a própria composição molar da entrada do estágio. A partir da integração numérica das Equações 3 e 4 de $\eta=0$ até $\eta=1$, pode-se simular diferentes cenários de operação. Neste trabalho, a resolução dos modos concorrente e contracorrente foi feita com o uso de pacotes numéricos de resolução de equações diferenciais do software Maple. Em cada modo operacional, procurou-se avaliar a dependência da vazão molar de $\mathrm{CO}_{2}$ em função do parâmetro $\zeta_{\mathrm{CO} 2}$, quando se fixa a composição desejada na saída do estágio. Tal análise foi desenvolvida para quatro diferentes composições de alimentação e duas diferentes razões de pressão (Casos A-D, Tabela 1) típicas do cenário previsto nas unidades de processamento do Pré-Sal brasileiro. Cabe ressaltar que, fixando-se as condições operacionais e a estrutura da membrana, o parâmetro $\zeta_{\mathrm{CO} 2}$ pode ser utilizado para avaliar a influência da vazão total molar, da pressão na corrente de concentrado (se fixado o $\varphi$ ) e da espessura da pele densa da membrana sobre a área de membrana requerida para se atingir uma determinada separação.

\subsection{Implementação em EMSO (diferentes configurações)}

O software EMSO () é um simulador que permite a inserção de equações específicas pelo usuário. Dentre suas principais características, é um resolvedor de equações algébricas, diferenciais, ou algébricodiferenciais, permitindo inclusive a introdução de pacotes de cálculo pelo usuário. 
Para a implementação em EMSO, as equações originais (1) e (2) (não adimensionadas) foram implementadas de forma discretizada, levando a um sistema algébrico. Foi adotado 20 intervalos de discretizações, valor para o qual se observou boa robustez e baixo desvio em relação à solução por outros métodos específicos para este fim. Embora o EMSO permita adaptações para o cálculo de equações diferenciais, a solução destas equações geralmente é empregada para sistemas dinâmicos (a variável independente é o tempo). Para o modelo estacionário consistindo de módulos de membranas com compressores e reciclos, tal adaptação não seria adequada, sendo esta a razão de empregar o modelo na forma discretizada. Foram empregados, além do modelo da membrana em forma discretizada, modelos de misturadores e compressores. No caso do bloco do compressor, em particular, implementou-se apenas um bloco de simulação que elevava a pressão da corrente à condição desejada, sendo mantida a mesma temperatura (operacionalmente teria de ser um ou mais compressores intercalados com trocadores de calor). Diferentes configurações foram investigadas, conforme ilustrado na Figura 2.
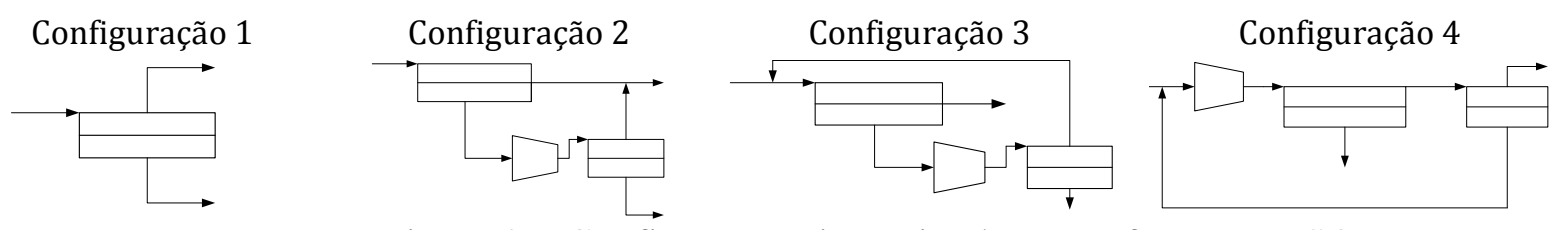

Figura 2 - Configurações investigadas no software EMSO.

\subsection{Condições investigadas}

Foram admitidos, com base em trabalhos da literatura (Baker e Lokhandwala, 2008), permeabilidade de $\mathrm{CO}_{2}$ de uma membrana a base de acetato de celulose de 70 Barrer, seletividade ideal de $\mathrm{CO}_{2}$ em relação ao metano de 15 , espessura da pele de $0,5 \mu \mathrm{m}$ e vazão total de entrada do concentrado de $1 \mathrm{~mol} / \mathrm{s}$ (base de cálculo). As condições de operação investigadas estão listadas na Tabela 1 e refletem condições de operação típicas de sistemas previstos no Pré-Sal (Nakao et al. 2009). Os casos A, B e C consideram variação na condição de alimentação (a vazão adimensional na entrada coincide com a fração molar das espécies), enquanto o caso D utiliza uma razão de pressão que o distingue dos demais.

Tabela 1 - Condições operacionais dos casos investigados.

\begin{tabular}{ccccc}
\hline & Caso A & Caso B & Caso C & Caso D \\
\hline Pressão do concentrado (bar) & 60 & 60 & 60 & 54 \\
Pressão do permeado (bar) & 1 & 1 & 1 & 4 \\
$\dot{v}_{\mathrm{CO} 2}^{\mathrm{c}}\left[\mathrm{z}_{0}\right]$ & 0,10 & 0,20 & 0,30 & 0,26 \\
$\dot{v}_{\mathrm{CH} 4}^{\mathrm{c}}\left[\mathrm{z}_{0}\right]$ & 0,90 & 0,80 & 0,70 & 0,74 \\
\hline
\end{tabular}

\section{RESULTADOS E DISCUSSÃO}

\subsection{Para único estágio}

Os resultados da simulação dos módulos co-corrente e contracorrente em único estágio estão apresentados na Figura 3 para os diferentes casos listados na Tabela 1. 


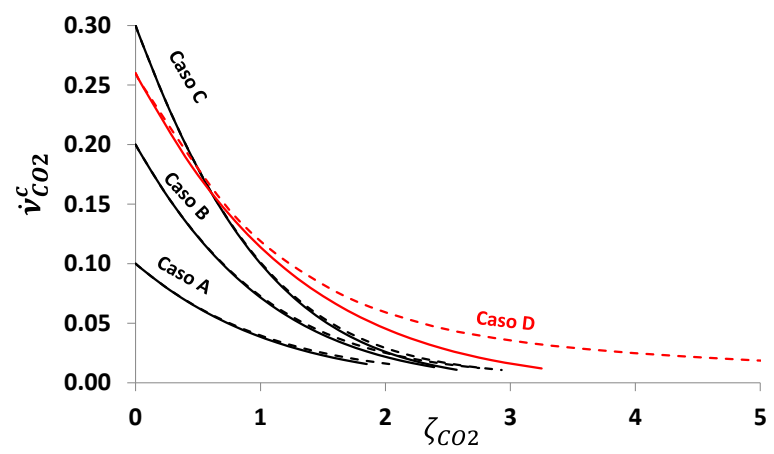

(a)

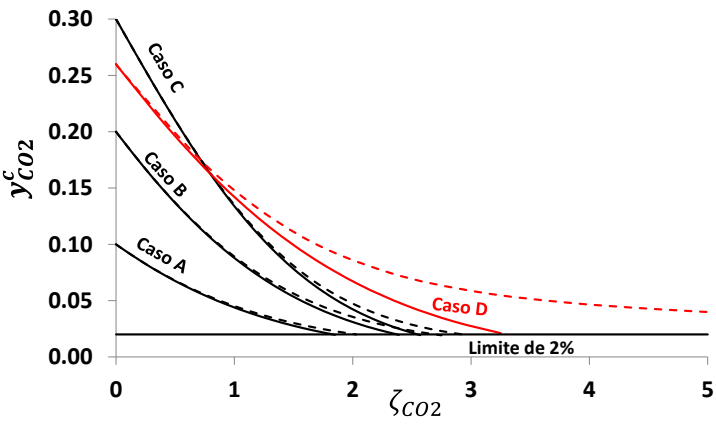

(b)

Figura 3 - Vazão adimensional (a) e composição de $\mathrm{CO}_{2}$ no concentrado (b) em função de $\zeta_{\mathrm{CO} 2}$ para um único módulo (-contracorrente, -- co-corrente ).

Para os casos A, B e C, os resultados da Figura 3 sugerem que os modos co-corrente e contracorrente só apresentam diferenças significativas quando se deseja maiores fatores de separação. O caso D é o mais discrepante dentre os demais, pois opera com uma razão de pressão aproximadamente 4,6 vezes menor que nos três primeiros. Nestas condições operacionais, são necessárias maiores áreas de membrana para atingir a concentração de $\mathrm{CO}_{2}$ de $2 \%$ no concentrado e as diferenças entre os modos de operação são bem mais pronunciadas (Tabela 2). Os valores de área da membrana por vazão total alimentada apresentados na Tabela 2 foram calculados utilizando as condições descritas no item 2.3. Como esperado, o desempenho do modo de operação em contracorrente foi superior ao modo co-corrente, pois exigiria menores custos de capital (áreas de membrana) para uma mesma especificação.

Segundo a abordagem adotada, os grupos adimensionais $\zeta_{i}$ e $\varphi$ definem a performance de operação do sistema por membranas e permitem estimar os custos de capital (área de membrana) adicionais, caso seja necessário aumentar a espessura da pele densa para suportar as pressões da corrente de alimentação no cenário do Pré-Sal. Adicionalmente, a partir do valor da variável $\zeta_{\mathrm{CO} 2}$ que permite atingir a especificação desejada, é possível ajustar a razão área/vazão mediante a manipulação de válvulas de controle, variando o número de módulos comerciais em série no estágio para atender a sazonalidade da operação de produção de gás natural no cenário do pré-sal.

Tabela 2 - Área por vazão total alimentada a partir de dados limite da Figura 3.

\begin{tabular}{ccccc}
\hline \multirow{2}{*}{ Modo de Operação } & \multicolumn{4}{c}{ Área da membrana por vazão total alimentada (s.m²/mol) } \\
\cline { 2 - 5 } & Caso A & Caso B & Caso C & Caso D \\
\hline Contracorrente & 6,5 & 8,4 & 9,0 & 11,6 \\
Concorrente & 7,1 & 9,6 & 10,3 & $>43$ \\
\hline Desvio percentual entre & $\approx 10 \%$ & $\approx 15 \%$ & $\approx 15 \%$ & $>270 \%$ \\
os modos de operação & & &
\end{tabular}

\subsection{Diferentes configurações dos módulos utilizando o EMSO}

Neste estudo, empregou-se o caso $\mathrm{C}$ da Tabela 1 operando em co-corrente, para uma fração de alimentação de $\mathrm{CO}_{2}$ na alimentação de $30 \%$, pior cenário dentre aqueles considerados. Aqui, $R_{A}$ denota a razão entre a área do segundo estágio e a área do primeiro 
estágio. Para as diferentes configurações dos módulos, de forma a se obter a concentração de $\mathrm{CO}_{2}$ de $2 \%$ na saída do sistema, investigou-se a dependência da área total de membrana por vazão alimentada e recuperação de metano em função de $R_{A}$ (Figura 4 a e b, respectivamente).

As configurações 1 e 4 atendem as especificações de composição de concentrado com a menor área total, entretanto fornecem as menores recuperações de metano. Os resultados apresentados na Figura 4 sugerem que todas as configurações com dois estágios apresentam uma área total mínima em relação à $R_{A}$. Entretanto, em termos de recuperação de metano, as configurações 2 e 3 apresentam tendências contrárias aquelas observadas para a configuração 4 , que fornece maiores valores a medida que se aumenta $R_{A}$.

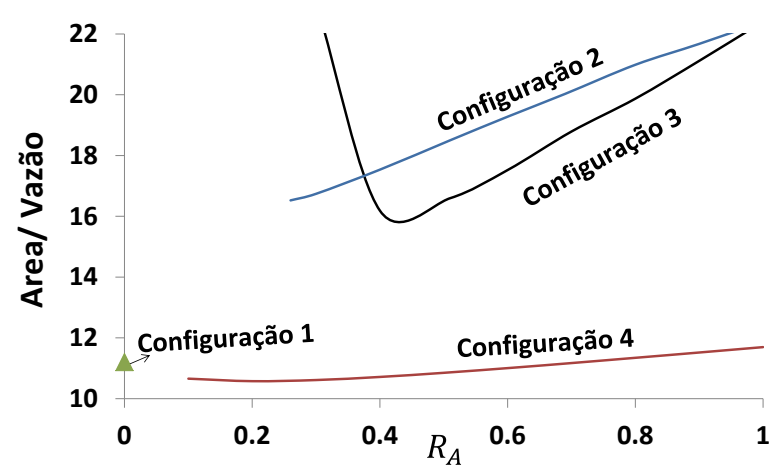

(a)

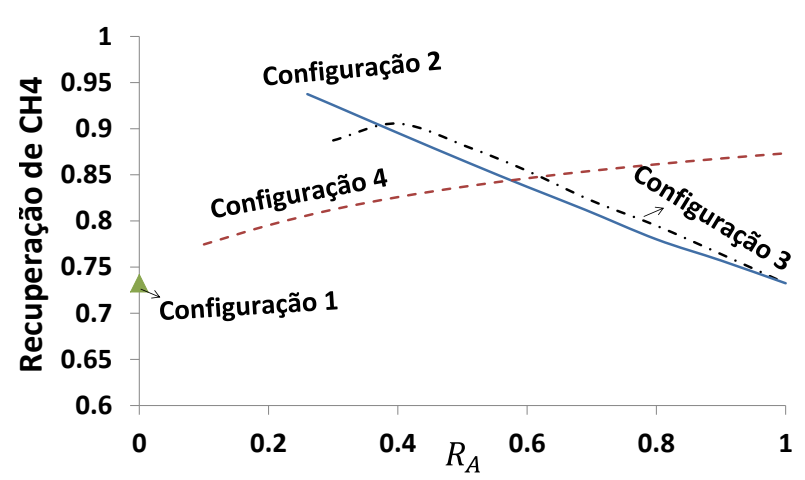

(b)

Figura 4 - Razão Área/Vazão alimentada (a) e recuperação de metano (b) em função de $R_{A}$ para diferentes configurações de estágios.

O cenário mais vantajoso é certamente identificado a partir de um critério econômico, como por exemplo, no trabalho de Gadelha et al. (2012). Contudo, é fundamental também avaliar as configurações do sistema de permeação para diferentes cenários econômicos. No trabalho de Deng et al. (2010), a configuração 3 foi identificada como ótima segundo os critérios adotados no tratamento de biogás, isto é, tal configuração mostrou-se vantajosa quando a recuperação de metano norteia o critério econômico. Por outro lado, o cenário do Pré-Sal brasileiro apresenta peculiaridades, pois a carga exigida nos compressores pode tornar etapas de reciclo de correntes do permeado economicamente inviável, além do fato da necessidade de elevadas vazões de gás reinjeção nos poços reduzirem a relevância de critérios econômicos baseados na recuperação de metano. O emprego de técnicas de otimização tornase necessário para avaliar a configurações e condições operacionais que levam ao máximo retorno econômico.

\section{CONCLUSÃO}

Neste trabalho, foi utilizado um modelo de transferência de massa em módulo de fibrasocas na avaliação diferentes configurações de sistemas com membranas, envolvendo etapas de re-compressão e reciclo, para remoção do $\mathrm{CO}_{2}$ do gás natural. Foram adotados na modelagem valores típicos de permeabilidade e seletividade em membranas comerciais de acetato de celulose e condições operacionais esperadas na produção de petróleo nos poços do Pré-Sal brasileiro. A partir de uma abordagem envolvendo grupos adimensionais, foi possível avaliar a influência do modo de operação e da composição de entrada sobre a área de membrana requerida e a recuperação de metano para atingir uma composição especificada na 
corrente de concentrado. Para ambas variáveis analisadas, o modo co-corrente apresenta resultados inferiores, principalmente para menores razões de pressão e teores de $\mathrm{CO}_{2}$ na corrente de concentrado. A abordagem utilizando grupos adimensionais mostra-se interessante, pois permite ajustar uma razão área/vazão em função da espessura da pele densa necessária para suportar as elevadas pressões previstas para as correntes de alimentação e da sazonalidade das composições desta corrente.

Neste estudo fica claro, que a configuração mais adequada dependerá do destino de cada corrente efluente do sistema de separação. No cenário do Pré-Sal brasileiro, estão previstas reinjeção de elevadas vazões de gás para a produção dos poços. Caso seja necessário vazões superiores às quantidades de $\mathrm{CO}_{2}$ produzidas no sistema de tratamento de gás natural, o foco será apenas a especificação da composição de concentrado com o menor custo de capital e operação. Neste cenário, os resultados deste trabalho apontam para a utilização da configuração 1 em contracorrente. Por outro lado, se a recuperação de metano nortear os critérios, este estudo aponta para a uma otimização associada a uma análise econômica.

\section{REFERÊNCIAS}

ENGELIEN, H. K., Process Integrated Membrane Separation - with Application to the Removal of $\mathrm{CO}_{2}$ from Natural Gas. Department of Chemical Engineering, NTNU, Nordisk Energiforskning, 2004.

DENG, L.; HÄGG, M. B. Techno-economic evaluation of biogas upgrading process using $\mathrm{CO}_{2}$ facilitated transport membrane. Int. J. Greenh. Gas Control, v. 4, p. 638-646 2010;

NAKAO, A.; MACEDO, A. P. F.; VERSIANI, B. M.; ARAÚJO, O. Q. F.; MEDEIROS, J. L. Modeling of flowcharts of permeation through membranes for removal of $\mathrm{CO}_{2}$ of natural gas. Proceedings of the 10th International Symposium on Process Systems Engineering, Salvador, Brasil, p. 1875-1880, 2009.

KUNDU, P. K., CHAKMA, A.; FENG, X. Modelling of multicomponent gas separation with asymmetric hollow fibre membranes - methane enrichment from biogas, Can. J. Chem. Eng., v. 91, p. 1092-1102, 2013.

BAKER, R. W.; LOKHANDWALA, K. Natural gas processing with membranes: an overview, Ind. Eng. Chem. Res. v. 47, p. 2109-2121, 2008.

GADELHA, T. S.; GUIMARÃES, A. R. S.; NAKAO, A.; ARAÚJO, O. Q. F.; MEDEIROS, J. L. A comparative economical analysis of technologies for $\mathrm{CO}_{2}$ removal from offshore natural gas, Proceedings of the 11th International Symposium on Process Systems Engineering, p. 1875-1880, Singapore, p. 800-804, 2012.

SCHOLES, C. A.; STEVENS, G. W.; KENTISH, S. E. Membrane gas separation applications in natural gas processing, Fuel, v. 96, p. 15-28, 2012.

YAVE, W.; CAR, A.; FUNARI, S. S.; NUNES, S. P.; PEINEMANN, K. V. CO2-philic polymer membrane with extremely high separation performance, Macromolecules, $\mathrm{v}$. 43, p. 326-333, 2010. 